\title{
Tai chi for treating osteopenia and primary osteoporosis: a meta-analysis and trial sequential analysis
}

\author{
Yili Zhang',2 \\ Yan $\mathrm{Chai}^{3}$ \\ Xiaojie $\mathrm{Pan}^{4}$ \\ Hao Shen' \\ Xu Wei ${ }^{5}$ \\ Yanming $\mathrm{Xie}^{1}$ \\ 'Institute of Basic Research in \\ Clinical Medicine, China Academy \\ of Chinese Medical Sciences, Beijing, \\ China; ${ }^{2} \mathrm{~S}$ Chool of Traditional Chinese \\ Medicine, Beijing University of \\ Chinese Medicine, Beijing, China; \\ ${ }^{3}$ Department of Epidemiology, \\ University of California, Los Angeles, \\ CA, USA; ${ }^{4}$ Department of Human \\ Nutrition and Health, Wageningen \\ University and Health, Wageningen, \\ The Netherlands; ${ }^{5}$ Department of \\ Scientific Research, Wangjing Hospital, \\ China Academy of Chinese Medical \\ Sciences, Beijing, China
}

This article was published in the following Dove Medical Press journal:
Clinical Interventions in Aging
Purpose: The aim of this meta-analysis was to evaluate the efficacy of Tai chi (TC) as an adjuvant treatment for osteopenia and primary osteoporosis.

Methods: We went through eight databases to identify relevant randomized controlled trials that compared TC with a control group. The primary outcome was osteoporosis-related fractures (fracture incidence). Meta-analyses and trial sequential analyses (TSA) were conducted using RevMan 5.3 and TSA 0.9.

Results: Fifteen randomized controlled trials involving a total of 857 patients were included in the analyses. No trials reported primary outcome; however, bone mineral density (BMD) values differed significantly in subgroup 1 (TC vs no treatment; weighted mean difference [WMD] $=0.05 \mathrm{~g} / \mathrm{cm}^{2}, 95 \%$ CI 0.03 to $0.07 ; P<0.00001 ; P$ for heterogeneity $=0.22, I^{2}=22 \%$ ) and subgroup 2 (TC vs conventional treatments; WMD $=0.16 \mathrm{~g} / \mathrm{cm}^{2}, 95 \% \mathrm{CI} 0.11$ to 0.21 ; $P<0.00001 ; P$ for heterogeneity $=0.008, I^{2}=75 \%$ ). In addition, two trials compared TC with conventional treatments, which found a significant difference in bone gla protein (standardized mean difference $=-1.18,95 \% \mathrm{CI}-1.66$ to $-0.70 ; P<0.00001 ; P$ for heterogeneity $=0.58$, $I^{2}=75 \%$ ). The results of the BMD were confirmed by TSA. Also, TC may have a certain effect on the relief of osteoporotic pain $(\mathrm{WMD}=-2.61,95 \% \mathrm{CI}-3.51$ to $-1.71 ; \mathrm{WMD}=-1.39,95 \%$ CI -2.01 to -0.77 ). However, it did not promote the quality of life, level of serum calcium, serum phosphorus, and also had no effect on bone turnover markers.

Conclusion: Although there is no study monitoring fracture incidence, TC may be beneficial for patients in improving BMD values, level of bone gla protein, and relieving osteoporotic pain. However, due to the low methodological quality, current evidence for treating osteopenia and primary osteoporosis through TC is insufficient.

Keywords: Tai chi, osteopenia, primary osteoporosis, evidence based medicine, trial sequential analyses

\section{Introduction}

Primary osteoporosis (POP) is a worldwide health problem with a consequent increase in bone fragility and susceptibility to fractures. ${ }^{1}$ It is characterized by low bone mass and microarchitectural deterioration of bone tissue. ${ }^{2,3}$ Osteopenia is a condition of decreased bone density and a precursor of osteoporosis. ${ }^{4}$ Osteoporosis affects about 200 million people worldwide and is a huge cost for the healthcare system. This cost is estimated to increase to $\$ 25.3$ billion per year. $^{5}$

Pharmacologic treatments for osteoporosis include bisphosphonates, estrogen, selective estrogen receptor modulators, and so forth. ${ }^{6-8}$ The aim of most of these treatments is to prevent bone resorption. Dietary supplements, like calcium and vitamin D, are also 
used as basic treatment. However, because of the necessity of lifelong treatment and the potential negative side effects, like upper gastrointestinal symptoms and risk of venous thrombosis, research for alternative treatments remains pertinent. ${ }^{9}$

Physical activity is an important lifestyle factor for growth, development, and sustained health throughout life. In recent years, the benefits of physical activity have drawn more attention to its physiological effects on the body. ${ }^{10}$ Considering the side effect of pharmacological treatment for osteoporosis, there is an increasing demand for nonpharmacologic therapy, such as physiotherapy and physical activity that are used appropriately and for long periods. ${ }^{11}$ Previous experiments and/or clinical trials based on different types of physical activity such as aerobic exercise and/or resistance exercise have provided fundamental knowledge on this topic. In several studies, it has been demonstrated that both in human and in animal models, there is an increased expression of osteoprotegerin (OPG) consequent to a training program. ${ }^{12}$ Exercise can also influence the secretion of some hormones involved in bone formation, such as parathyroid hormone, prostaglandin E2, and estrogen. ${ }^{13}$ Moreover, some authors reported higher levels of anti-inflammatory cytokines such as IL-2 and IL-10, and lower levels of proinflammatory cytokines such as TNF- $\alpha$ and IL-6, as a consequence of moderate exercise, suggesting that exercise can also promote bone formation and inhibit bone resorption by OPG/RANKL/ RANK-independent signaling pathways. ${ }^{14}$ In addition to training programs, many studies support the effectiveness of the whole-body vibration training, and in particular, its positive effects on bone turnover in osteoporosis. ${ }^{15-17}$

Tai chi (TC) is a traditional systematic physical activity, which is widely practiced not only in China, but also has a large number of trainers worldwide. ${ }^{18,19} \mathrm{TC}$ employs precise regimens of physical movement, breathing techniques, and cognitive exercises (both visualization and focused internal awareness) to strengthen, relax, and adjust physical functioning and mental health. ${ }^{20-22}$ Over the past 25 years, TC has grown in popularity and gained worldwide recognition for its health benefits. Aside from pharmacological methods, muscle strengthening and weight-bearing exercises, which prevent osteoporosis by increasing bone and muscle strength as well as coordination and balance, TC is often recommended to patients in addition to bisphosphonates administration. Previous studies have also focused on finding evidence of the effects of TC on pain relief and physical improvements in patients with illness like knee osteoarthritis and fibromyalgia. ${ }^{23-25}$

Moreover, clinical trials assessing TC treatment for osteopenia and osteoporosis have recently been published and may have strengthened the evidence base that TC is one of the nonpharmacological therapy treatments that are effective. However, treatment of osteopenia and POP by TC is not fully summarized based on the latest evidence. Therefore, we conducted an updated meta-analyses of randomized controlled trials (RCTs) to investigate the efficacy of TC treatment for regulating the bone mass, especially for the patients with osteopenia and POP and those at high risk of osteoporosis. Furthermore, we examined whether the current evidence was robust and conclusive by using trial sequential analyses (TSA).

\section{Materials and methods Eligibility criteria}

This meta-analyses was reported in accordance with Preferred Reporting Items for Systematic Review and MetaAnalyses. ${ }^{26}$ All the included studies would be critically appraised by using the tool from the Cochrane Handbook for Systematic Reviews of Interventions. ${ }^{27}$ The protocol of this systematic review has been registered on PROSPERO (http://www.crd.york.ac.uk/prospero) on September 6, 2017 (registration number: CRD42017074663).

\section{Types of studies}

Only RCTs were included. There were no restrictions on language and setting. Quasi-experimental studies, animal experiments, duplicate studies, and studies for which the full text was not available to us were excluded. Review articles, case reports, editorials, letters, and comments were also excluded.

\section{Types of participants}

In this review, we paid close attention to the TC exercises for regulating the bone mass. The diseases we mainly focused on were osteopenia and POP so that the participants with osteopenia and POP or those at high risk of osteoporosis (mean age $>50$ years old) were included. The clinical diagnosis for osteopenia or POP should be in accordance with recognized international criteria, for instance $\mathrm{WHO}$ criteria: bone mineral density (BMD) of subjects, T-score $\leq-2.5$ could be defined as osteoporosis and $\mathrm{T}$-scores in the range of -2.5 and -1 could be defined as osteopenia. ${ }^{28}$ In addition, Chinese criteria (peak bone mass of subjects [mean \pm standard deviation] $>\mathrm{M}-1 \mathrm{SD} \sim 2 \mathrm{SD}$ could be defined as osteopenia and $<\mathrm{M}-2 \mathrm{SD}$ could be diagnosed as osteoporosis) were included as well. ${ }^{29}$

\section{Types of interventions}

In order to estimate the specific effect of TC, only the studies that had the TC as an intervention method were included. 
TC compared with a control group including no intervention, placebo, and conventional treatments or TC plus conventional treatments compared with conventional monotherapy were taken into account. No limitation was imposed on the style of $\mathrm{TC}$, the times of exercise, and the duration of the treatment.

\section{Types of comparisons}

In the control group, only placebo, no treatments, or conventional treatments recommended by the guidelines or internationally recognized treatments were included. Conventional treatments were mainly recommended by national guidelines, such as Clinical guidelines for diagnosis and treatment of postmenopausal osteoporosis (AACE/ ACE) ${ }^{30}$ Clinician's Guide to Prevention and Treatment of Osteoporosis (NOF), ${ }^{31}$ and Guideline for diagnosis and treatment of primary osteoporosis (CSOBMR). ${ }^{32}$ Usually, calcium and vitamin $\mathrm{D}$ supplements are also considered as conventional treatment. Other complementary and alternative treatments (eg, osteoporosis bone protective medication, physiotherapy, osteopathy, orthoses, herbal medicine, acupuncture, moxibustion, massage, yoga) have been excluded.

\section{Types of outcomes}

Osteoporosis-related fractures (fracture incidence) were the primary outcomes that we followed with interest. The secondary outcomes included changes in BMD value, serum calcium, serum phosphorus, and bone gla protein (BGP). Biochemical markers of bone turnover type I collagen carboxyterminal peptide (CTX), quality of life,,$^{33}$ and recognized pain scales were also included as the secondary outcomes.

\section{Information sources and search strategy}

A comprehensive search strategy was carried out including PubMed (1950 to September 2017), EMBASE (1974 to September 2017), the Cochrane Library (1996 to September 2017), ClinicalTrials.gov (from inception to September 2017), China Knowledge Resource Integrated Database (1979 to September 2017), Chinese Science and Technique Journals Database (1989 to September 2017), Wan Fang Database (1990 to September 2017), and the Chinese Biomedical Database (1990 to September 2017). The reference lists of studies meeting the inclusion criteria were analyzed to identify additional relevant studies.

The following search terms were used in separate or combined ways: "osteoporosis"; "primary osteoporosis"; "postmenopausal osteoporosis"; "senile osteoporosis"; "osteopenia"; "bone loss"; "Tai chi"; "Tai ji"; "Tai chichuan"; "Shadow Boxing"; "clinical trial". There were no restrictions on language, the types of publications, and participants' characteristics. Literature was managed by using Note Express 3.2.0 software.

\section{Study selection}

Two independent reviewers scanned the retrieved studies and decided whether or not to continue further analyses based on three criteria: titles, abstracts, and keywords. If the information met the inclusion criteria, full articles were retrieved for further assessment. We retrieved full content of articles if there was any doubt about these criteria from the information given in the title and abstract. Authors were contacted to obtain relevant missing data if necessary and where resources allowed. Any disagreements were settled by third party.

\section{Data collection process}

Data concerning details of participants, interventions, comparisons, and outcomes were extracted independently by two reviewers. The data extraction form included the following items: 1) general information: title, authors, and year of publication; 2) population: sample size, age, diagnostic criteria; 3 ) interventions: dose, duration, and frequency; and 4) outcomes: outcomes specified above.

\section{Risk of bias in individual study}

The methodological quality of RCTs was assessed independently using criteria from the Cochrane Handbook for Systematic Review of Interventions, Version 5.1.0. ${ }^{27}$ Seven domains were considered, such as sequence generation (selection bias), allocation concealment (selection bias), blinding of participants and personnel (performance bias), blinding of outcome assessment (detection bias), incomplete outcome data (attrition bias), selective outcome reporting (reporting bias), and other biases. Three levels of "low risk", "high risk", or "unclear risk" marked the quality appraisal category. Any disagreements were resolved by mutual consensus.

\section{Data analysis}

Revman 5.3 software provided by the Cochrane Collaboration was used for the data analysis. All pooled outcome measures were determined using random effect models. For the dichotomous outcome, the pooled RR with 95\% CI was used as the measure of effect. For the continuous outcomes with same units, weighted mean difference (WMD) was used when the units of outcomes were consistent, while standardize mean difference was performed as the effect measure if the units of the outcomes were different. If the number 
of included studies was less than two or heterogeneity was apparent, meta-analysis was neither appropriate nor possible. Under these circumstances, the results of our systematic review were narratively reported. If the included studies had multiple arms, we identified the relevant intervention and control groups, and then combined the relevant groups into a single group before synthesizing the data.

\section{Subgroup analysis and sensitivity analysis}

Subgroup analysis was deemed necessary. In order to explore the potential sources of heterogeneity of the methodology, statistics, and clinical characteristics, sensitivity analyses were performed. When the results of the clinical trials varied widely and heterogeneity tests showed significant differences, we removed one trial that was significantly different from the other trials and then pooled the remaining studies to compare the results before and after.

\section{Confidence in cumulative estimate}

The quality of the evidence would be assessed by the GRADE tool. ${ }^{34,35}$ High-quality evidence was considered as RCTs with low risk of bias, which could produce direct and precise results for the clinical outcome. ${ }^{36}$ Based on five key domains (methodology quality, directness of evidence, heterogeneity, precision of effect estimates, and risk of publication bias), levels of quality of evidence were defined as high, moderate, low, and very low. ${ }^{37}$

\section{Trial sequential analysis}

TSA was conducted to obtain the primary result. Cumulative meta-analysis might result in false-positive results (type I error) because of an increased risk of random error from sparse data and repeated significance testing. ${ }^{38}$ TSA could control the $P$-value and widen the confidence intervals. ${ }^{39}$ Estimation of the required information size and trial sequential monitoring boundaries were the concepts and rationale combined by TSA. If the cumulative $Z$ curve entered the futility area or crossed the trial sequential monitoring boundary, the anticipated intervention effect might reach a sufficient level of evidence, and further trials would not be necessary.

We calculated the required information size based on mean difference (Empirical) and variance (Empirical) in change of BMD value. The type I error $(\alpha)$ and power $(1-\beta)$ were set as 0.05 and 0.80 , respectively. The TSA was conducted with the use of TSA version 0.9 beta software (http://www.ctu.dk/tsa).

\section{Results Study selection}

In total, 785 records were identified. After removing 270 duplicates among different databases, the remaining 515 records were screened further by reading the title and abstract. Then, we excluded 436 records that did not meet the eligible criteria in title or abstract and the full texts of remaining 79 records were downloaded for careful assessment. Finally, 64 articles were excluded upon further scrutiny for non-RCTs $(n=19)$; intervention measures did not meet the requirement of this review $(n=11)$, participants were not suitable $(n=12)$, study objectives different from the aim of this review $(n=6)$, protocols $(n=5)$, abstract $(n=2)$, and review articles $(n=9)$. There were 15 trials included in the review of which three trials ${ }^{41,47,50}$ were published in English and the others $^{40,42-46,48,49,51-54}$ in Chinese. The detailed process of search and identification is shown in Figure 1.

\section{Study characteristics}

All trials included were conducted by a parallel design. The duration of trials ranged from 4 months to 12 months while only one trial did not report the duration. ${ }^{45}$ Sample size was within the scope of 24-110, with a total of 857 participants included in this review. All included trials reported the outcomes we focused on and 14 trials referred to BMD values or index of quantitative ultrasound. Details of the 15 included trials are listed for characteristics of included trials (Table 1).

\section{Methodological quality}

There are five trials using random number table or other ways. Only two trials mentioned the allocation concealment through sealed envelope. Only one study protocol ${ }^{50}$ was available at ClinicalTrials.gov. The quality criteria related to blinding of patients was not satisfactory. However, it should be noted that blinding patients or performers is difficult to realize in trials involving exercise as intervention. ${ }^{55}$ Furthermore, three trials reported the dropout or withdrawal of patients. We also considered all included trials with "unclear" for the other bias, such as the sample size (Figure 2).

\section{The effects of therapy}

According to the different treatments, the studies were divided into three subgroups: TC vs no treatment (subgroup 1), TC vs conventional treatments (subgroup 2), and TC plus conventional treatments vs conventional treatments (subgroup 3). Among these subgroups, we assessed the primary and secondary outcomes separately for all participants (Table 2). 


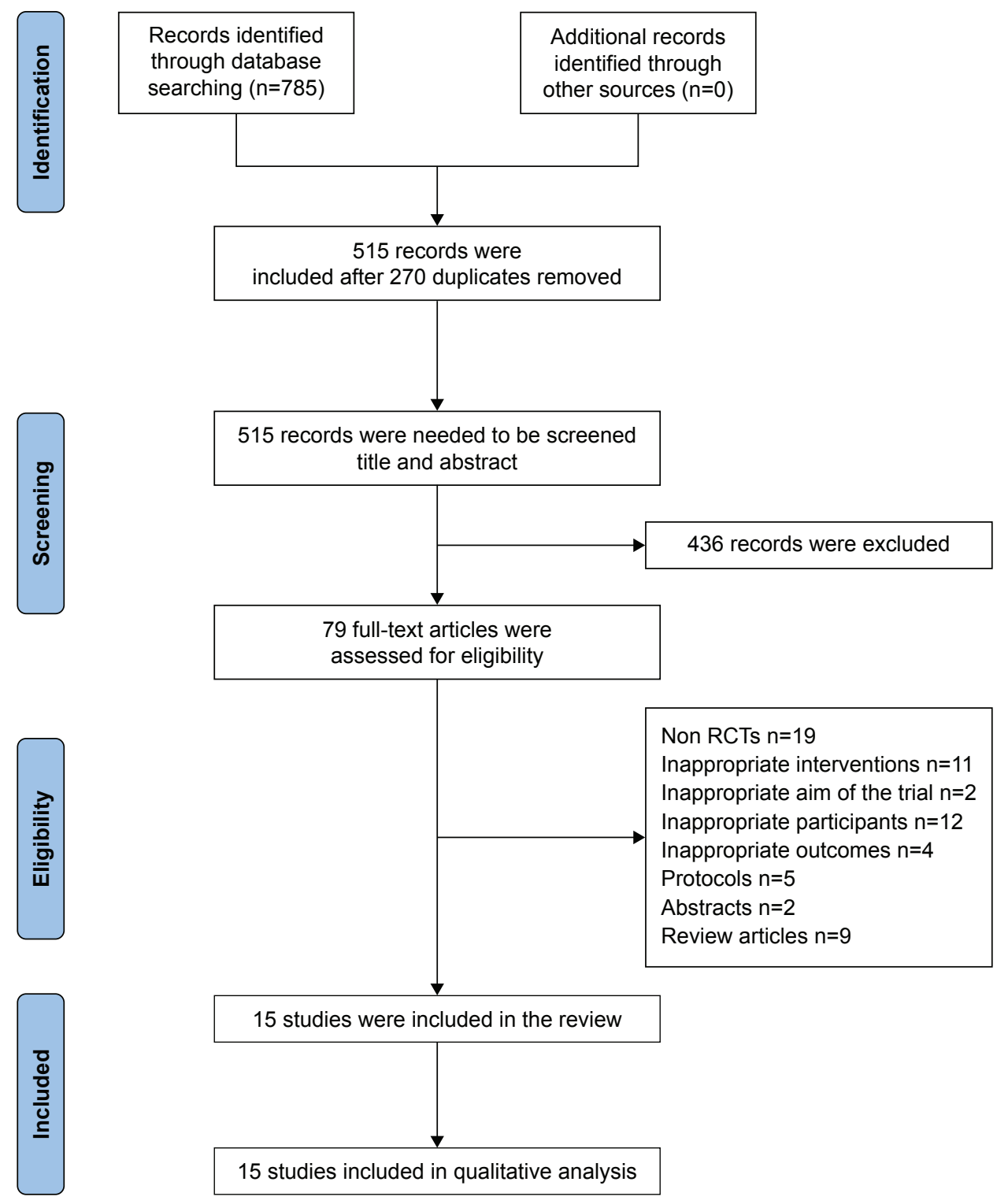

Figure I PRISMA flow diagram.

Abbreviations: PRISMA, Preferred Reporting Items for Systematic review and Meta-Analysis; RCTs, randomized controlled trials.

In addition, in order to explore the efficacy of TC in the treatment of osteopenia and POP, we conducted a separate analysis based on the included studies in which participants had confirmed osteopenia and POP (Table 3).

Although there is no study reporting primary outcome and no significant difference was detected in subgroup 3 on any outcomes, there was significant difference in BMD value in subgroup $1\left(\mathrm{WMD}=0.05 \mathrm{~g} / \mathrm{cm}^{2}, 95 \%\right.$ CI 0.03 to $0.07 ; P<0.00001 ; P$ for heterogeneity $=0.22, I^{2}=22 \%$ ) and subgroup 2 (WMD $=0.16 \mathrm{~g} / \mathrm{cm}^{2}, 95 \%$ CI 0.11 to 0.21 ; $P<0.00001 ; P$ for heterogeneity $=0.008, I^{2}=75 \%$ ) which indicates that TC has a positive effect on BMD value and the therapeutic effect is higher than conventional treatments. TSA showed that the cumulative $Z$ curves crossed both the conventional and the trial sequential monitoring boundaries and reached the significant area. Thus, it might be unlikely that further trials would change the conclusion (Figure 3). In addition, two trials compared $\mathrm{TC}$ with conventional treatments on BGP, which found a remarkable difference between the two groups (MD $=-1.18,95 \% \mathrm{CI}-1.66$ to -0.70 ; $P<0.00001 ; P$ for heterogeneity $=0.58, I^{2}=75 \%$ ).

Meanwhile, TC was beneficial to the level of BGP and relieved patient's pain (Visual Analog Scale). However, TC did not promote the quality of life and levels of serum 


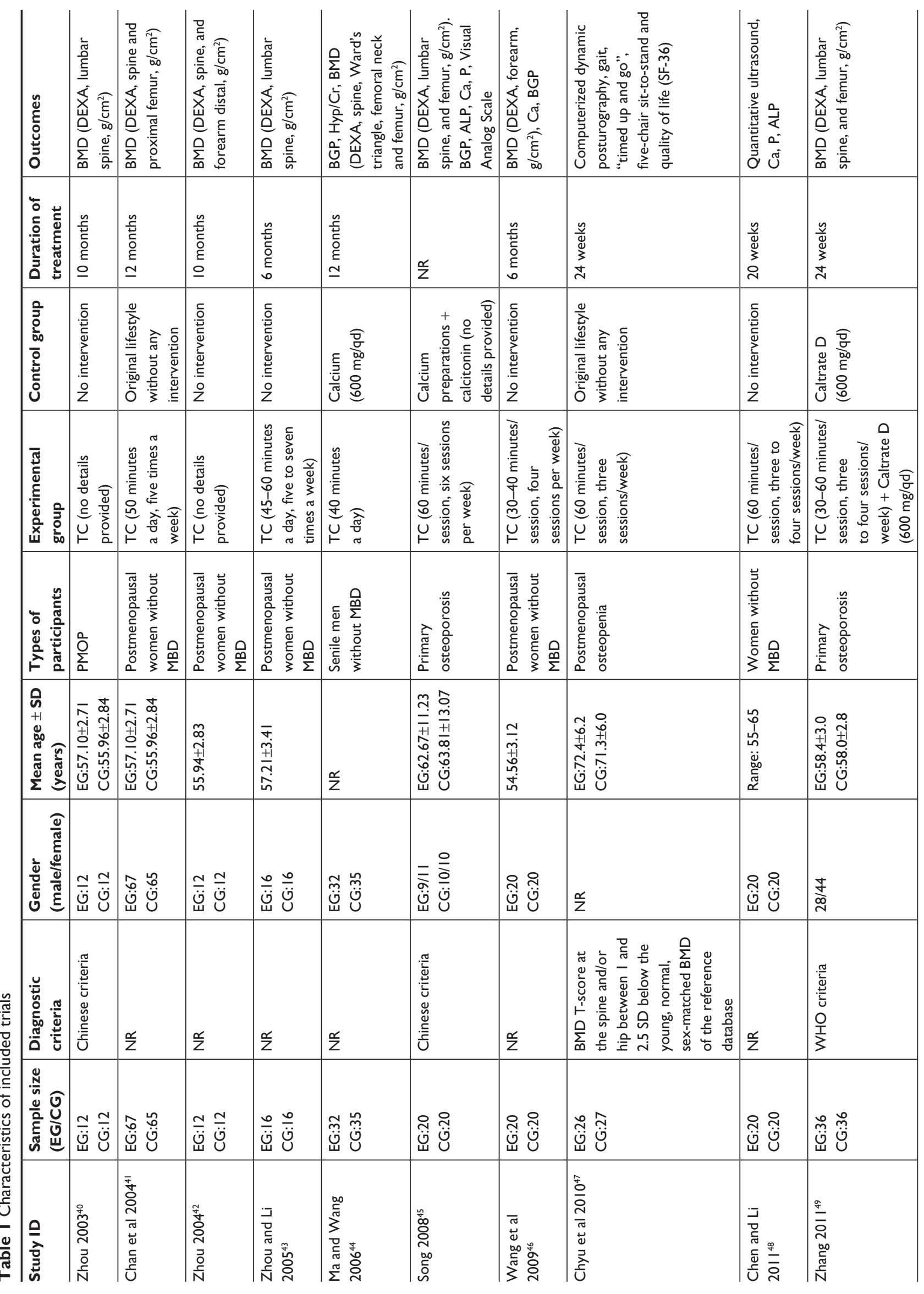




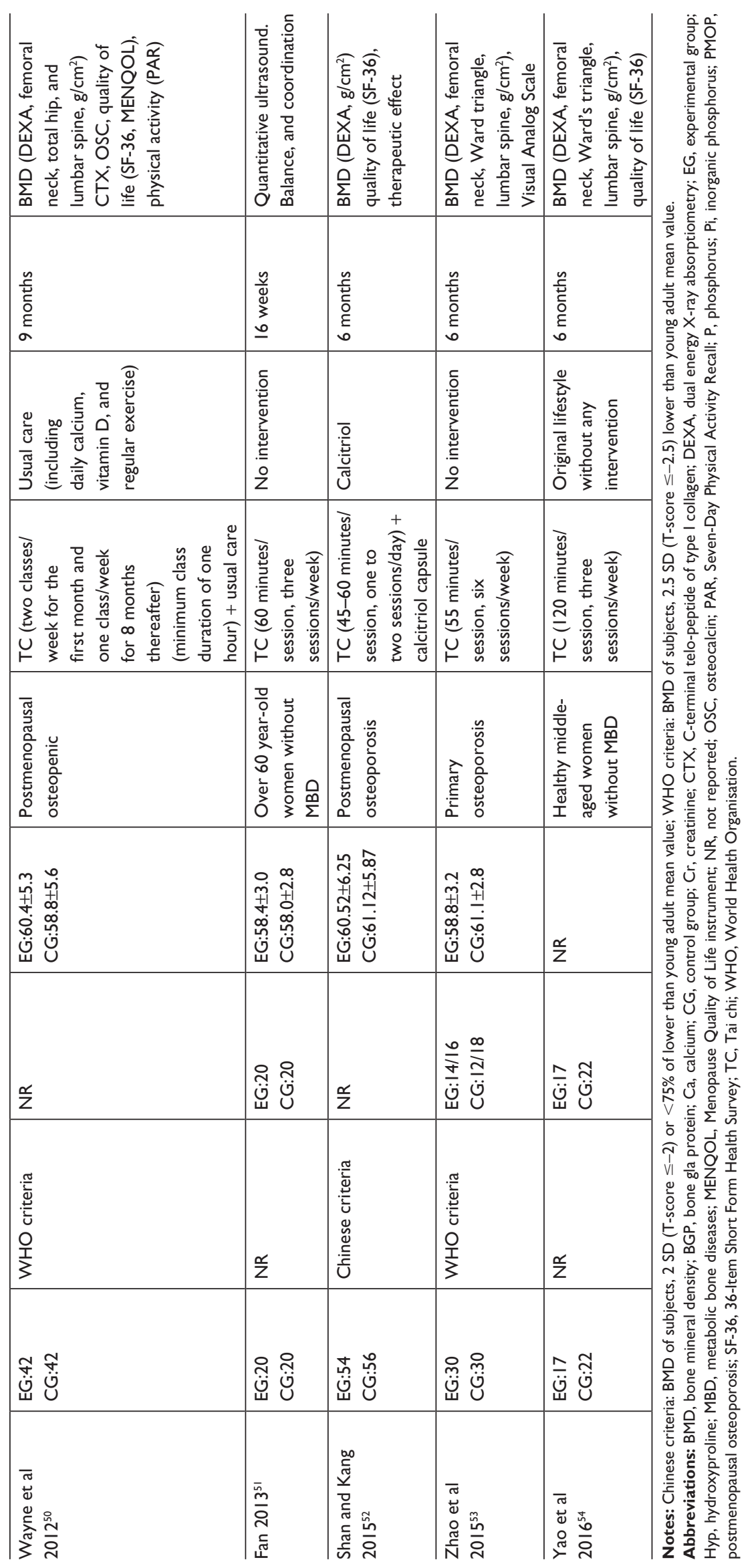




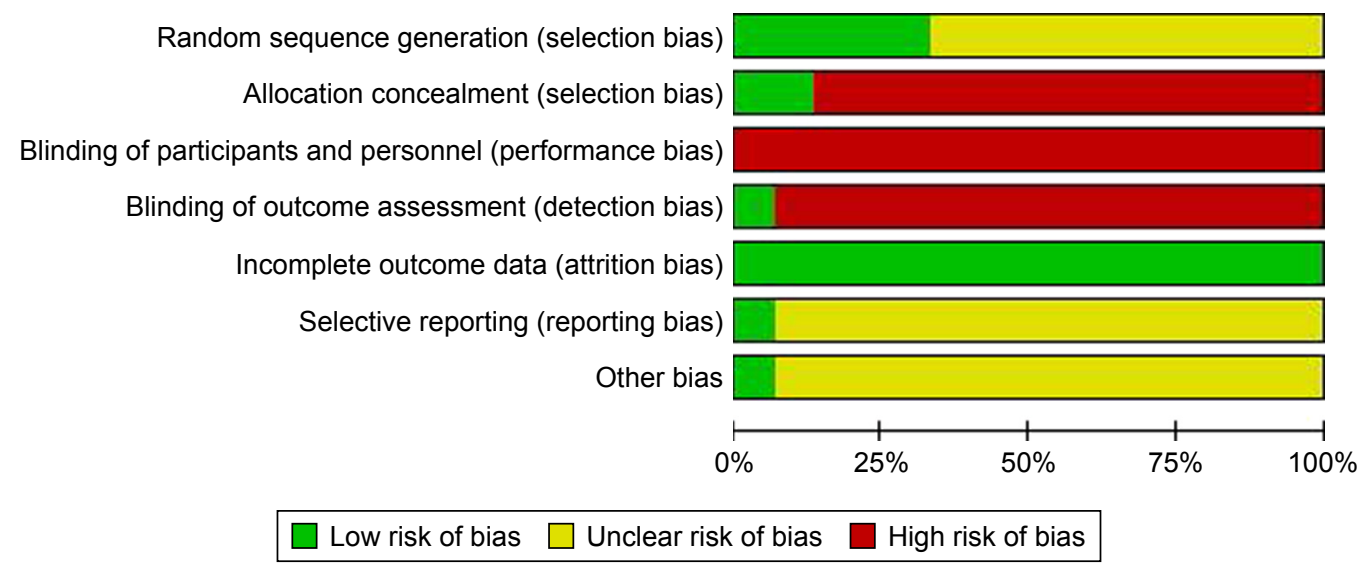

Figure 2 Risk of bias graph.

calcium, and serum phosphorus. According to the analysis, TC had no effect on CTX and osteocalcin.

\section{Funnel plot analysis}

Funnel plot analysis could not be conducted due to the small number of included studies $(<10)$ for the same outcome in the meta-analysis.

\section{Overall quality of evidence by GRADE}

We graded the overall quality of available evidence with the GRADE approach. The quality of evidence for all outcomes was downgraded to "low" or "very low" mainly due to high risk of performance bias and imprecision (small number of total events or small sample size).

\section{Discussion}

This systematic review and meta-analysis, which was based on 15 RCTs including 857 participants, found that TC may have a positive effect on BMD values, both as a single treatment and in combination with others. The evidence was validated by TSA. In order to better guide clinical practice, we assessed the achieved evidence of the GRADE approach. Due to the low quality of included trials and the above outcomes from individual trials, we could not draw firm conclusions from current evidence. Thus, further research is very likely to have an important impact on our confidence in the estimate of effect and is likely to change the estimate.

Three previous meta-analyses ${ }^{56-58}$ have reported on the same topic, as presented in Table 4. Differences between the present meta-analyses and the previous ones are as follows: firstly, our analyses included four additional trials that were published in the past 2 years. As the latest and most comprehensively updated meta-analyses, the present study further reinforces the results of previous meta-analyses. Second, we focused on more representative and more specific outcomes, which might fully describe the effect of TC on patients with osteopenia and POP and strengthened the current body of evidence. Third, we registered the protocol of this study on PROSPERO. A registered protocol may increase the transparency and quality of meta-analyses. Finally, TSA was further applied to estimate the effect more conservatively.

Based on currently available literature, there are some limitations due to the flaws of design, measurement, and evaluation in original studies. First, the included RCTs in this meta-analysis were performed in different patient groups and various clinical settings. The risk of potential heterogeneity, therefore, is present. Second, TC is based on traditional exercises which could not be blinded for physicians and patients so that a performance bias may have been introduced. Meanwhile, the problem with the lack of blinded outcome assessment may have also introduced information bias. Third, patients may take a long time to practice TC in order to achieve the purpose of the treatment, but the trials included in this study were generally short in length of duration. This may reduce the apparent effectiveness of TC. Fourth, this study focused only on the efficacy of TC on regulating bone mass, though the safety or the impact of TC on muscle strength, functional mobility, balance, and coordination or fracture incidence are important. In addition, complete and transparent reporting in quality and methodology should be in accordance with well-known standards for further research. At the same time, different types of TC may produce different outcomes. The style of TC should be clearly defined in the trials. Participants that withdrew/dropped-out during the trials should be clearly described and intention-to-treat analysis could be performed in data analysis.

Previous studies indicated that vitamin D and calcium supplementation are the most widely used therapies for 


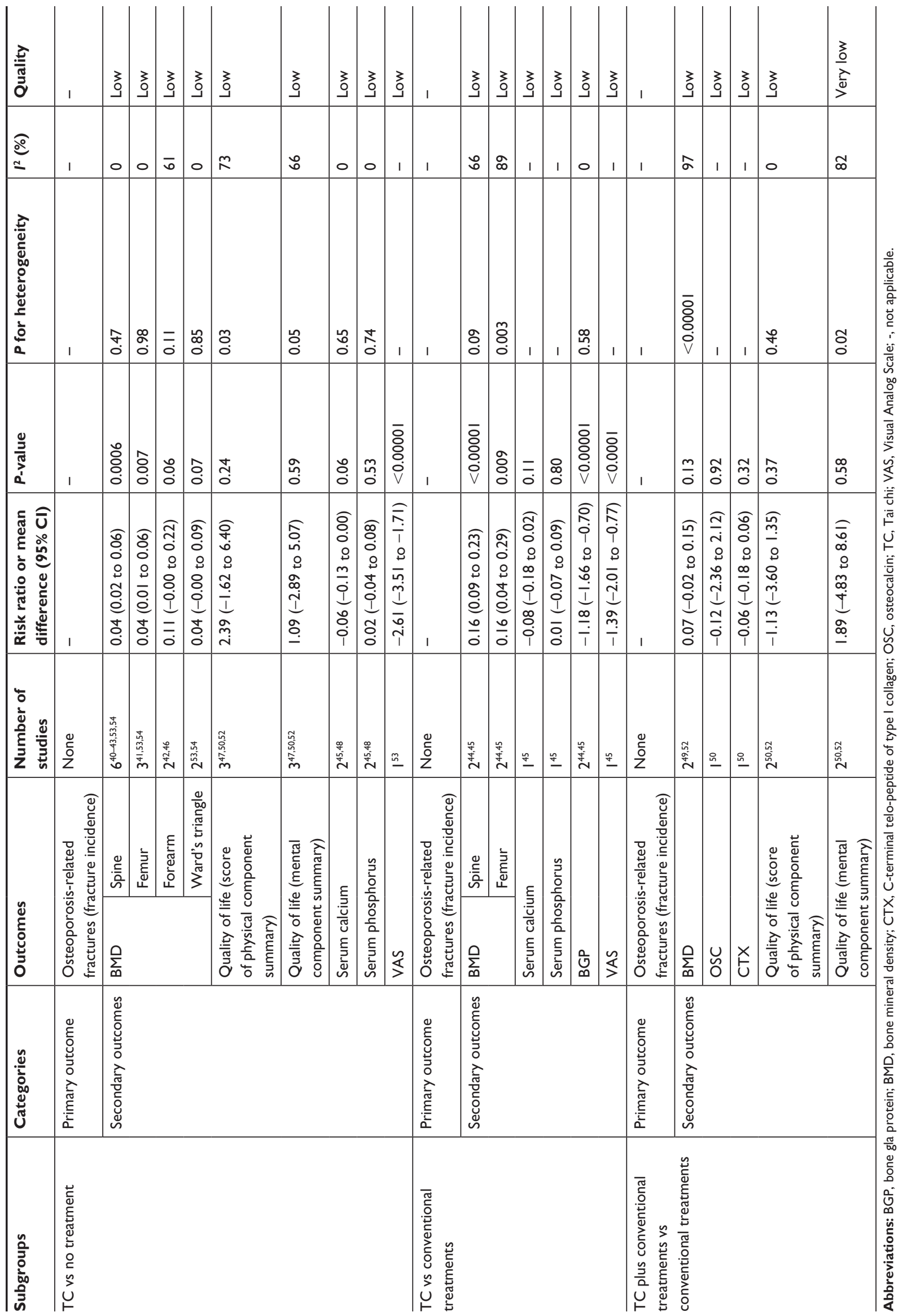




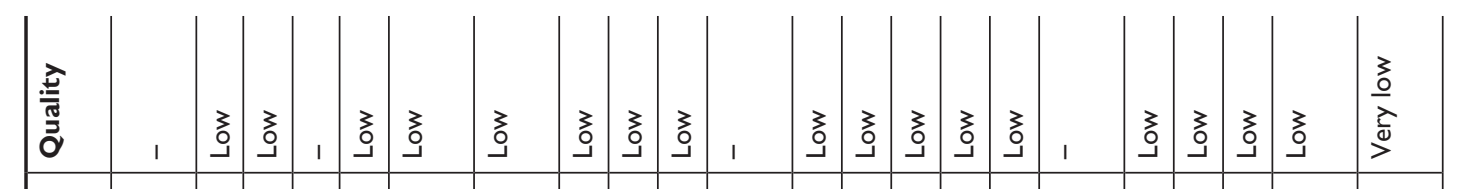

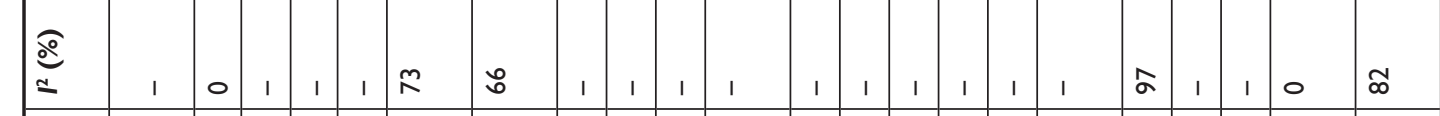

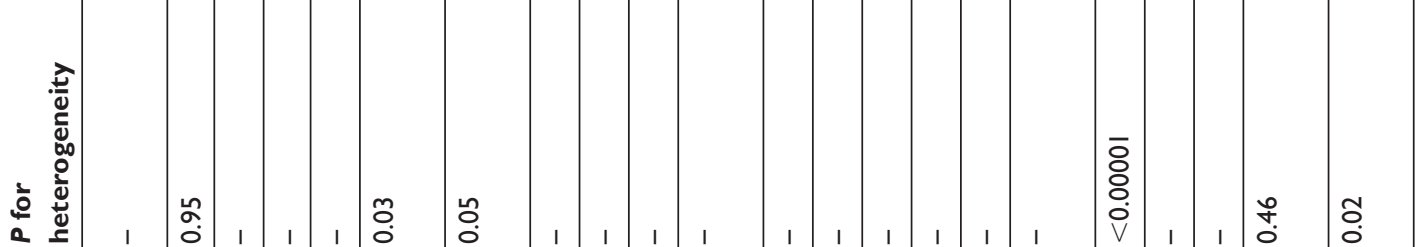

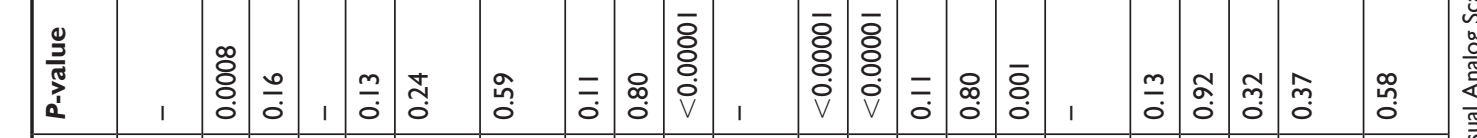

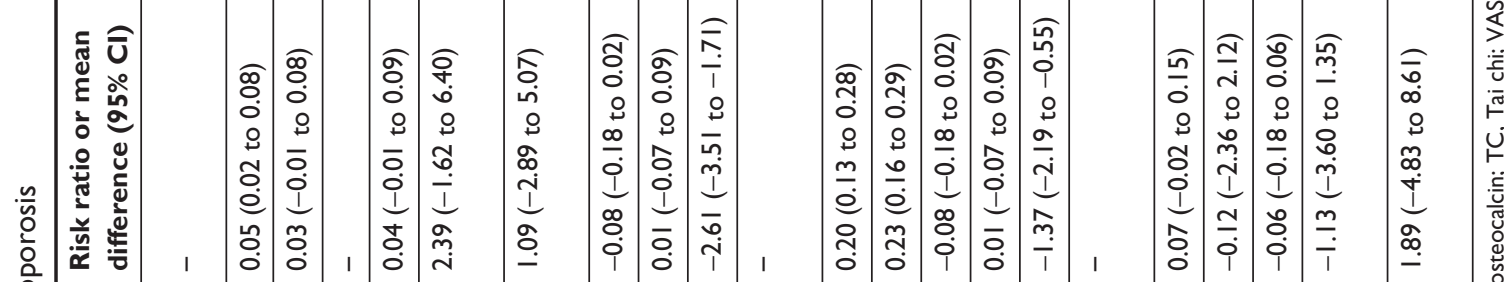

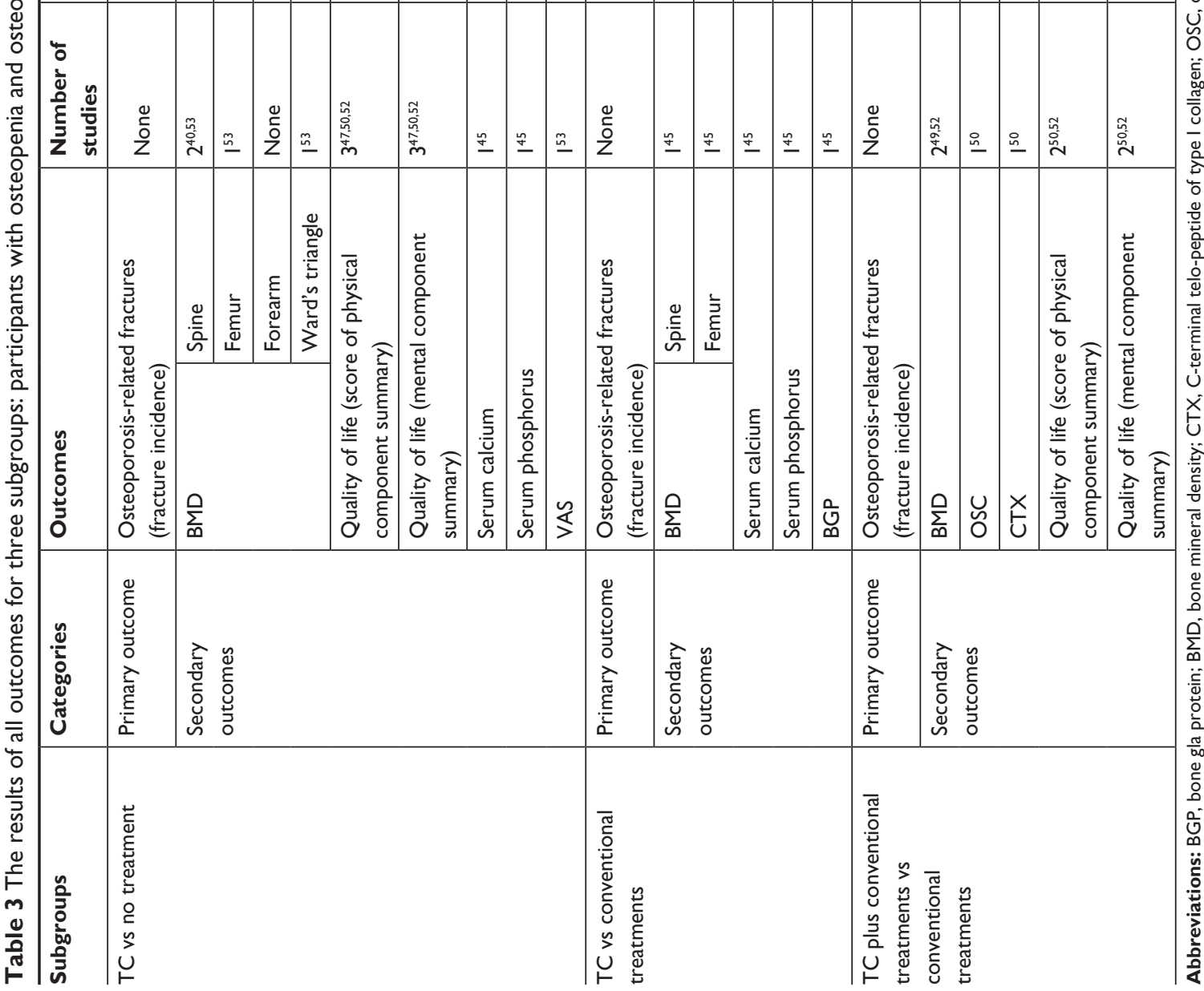




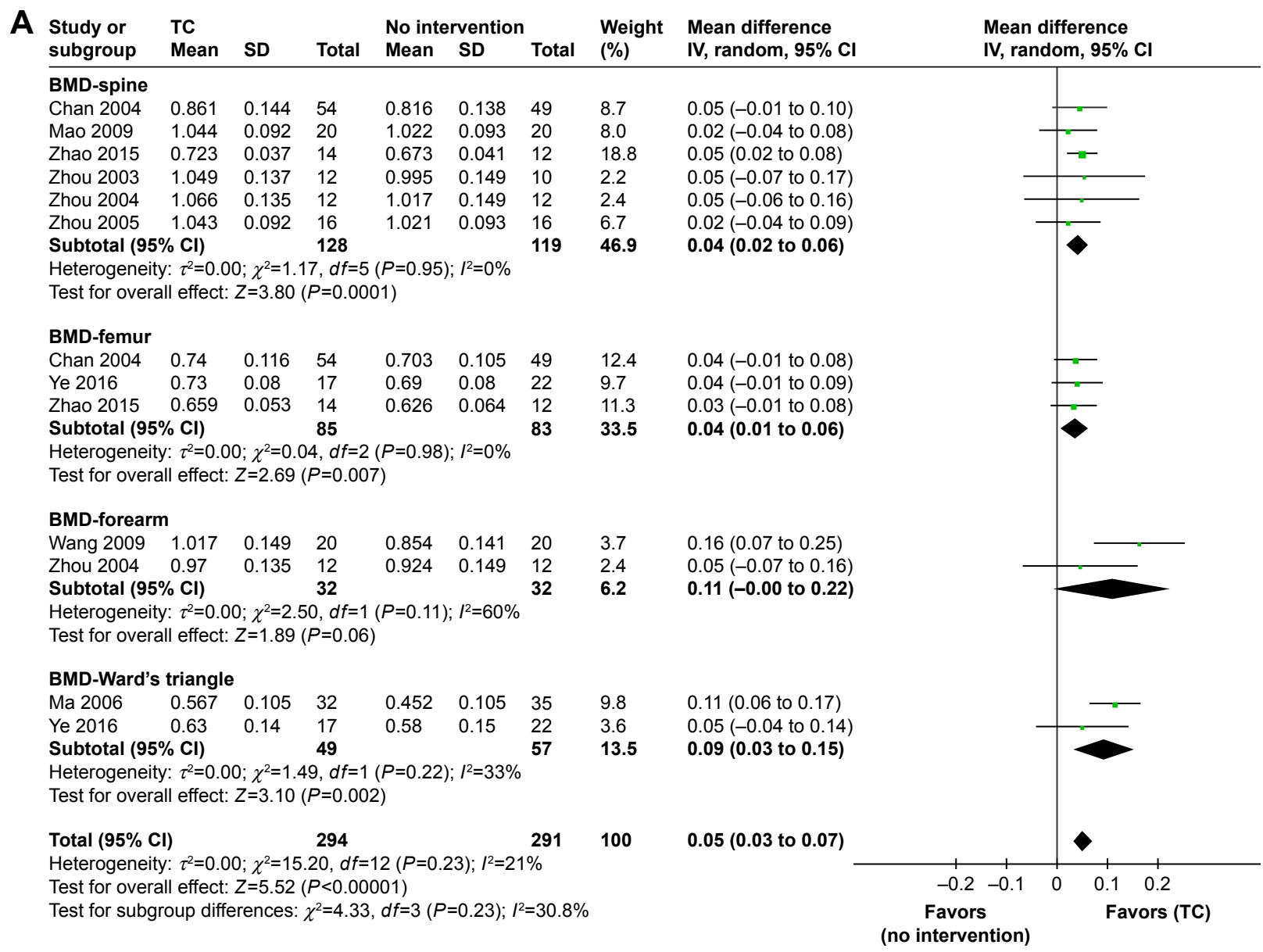

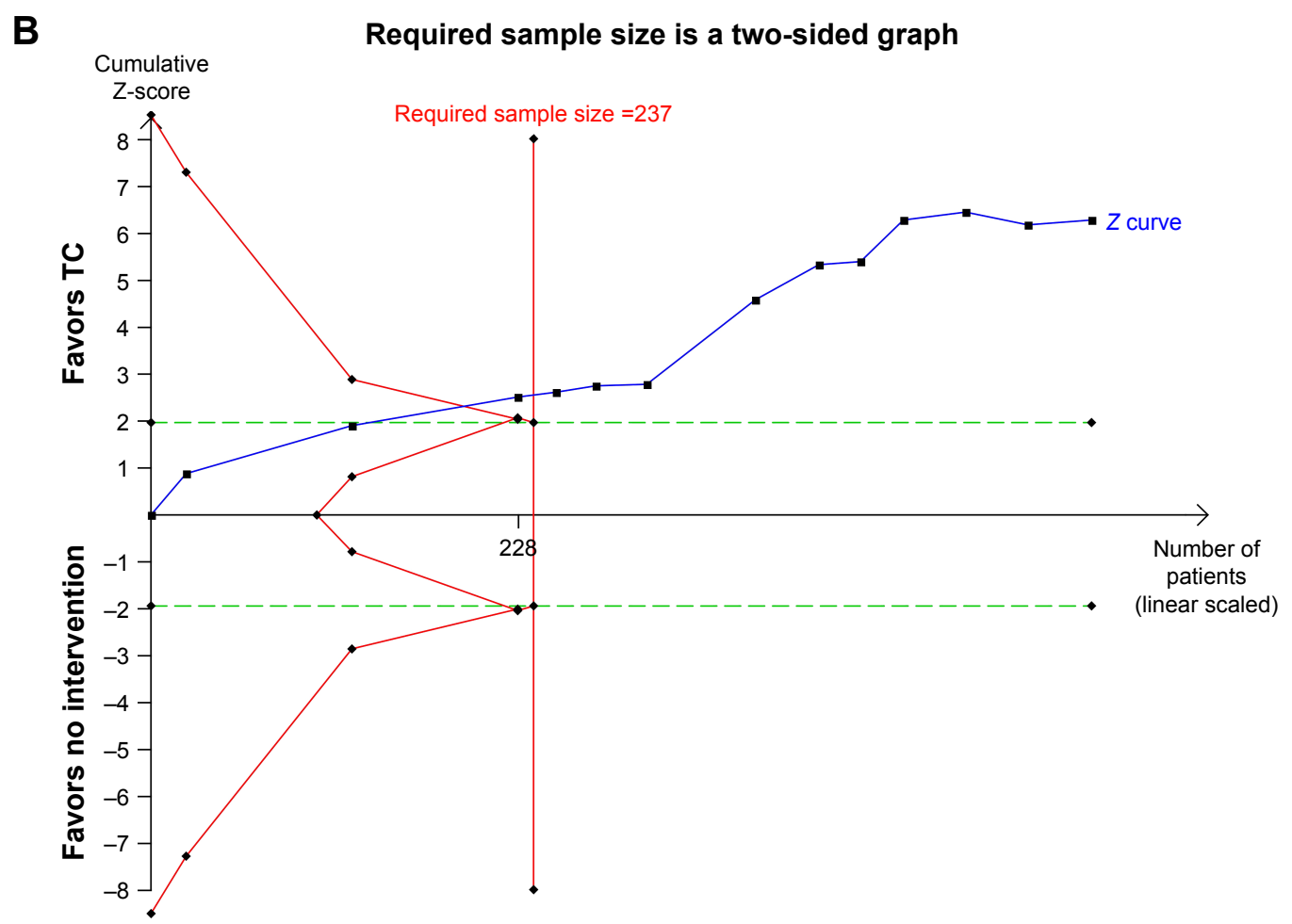

Figure 3 (A) TC vs no intervention: BMD value (all participants). (B) TSA for BMD value (TC vs no intervention) with an $\alpha$ of $5 \%$ (two-sided) and $\beta$ of $20 \%$. Notes: The required information size was calculated as 237. $Z$ curve has across-trial sequential monitoring boundary for benefit (all participants).

Abbreviations: BMD, bone mineral density; IV, inverse variance; TC, Tai chi; TSA, trial sequential analysis. 
Table 4 Comparison with other previous meta-analyses

\begin{tabular}{l|l|l|l|l}
\hline Author & Lee et al $\mathbf{2 0 0 8}^{\mathbf{5 6}}$ & $\mathbf{X u}$ and Liu 20 I $\mathbf{2}^{\mathbf{5 7}}$ & Sun et al 20 $\mathbf{6}^{\mathbf{5 8}}$ & The present meta-analysis \\
\hline Number of RCTs & 5 & 5 & $\mathrm{II}$ & $\mathbf{1 5}$ \\
\hline Participants & Osteoporosis & $\begin{array}{l}\text { Postmenopausal } \\
\text { women }\end{array}$ & $\begin{array}{l}\text { Perimenopausal and } \\
\text { postmenopausal women }\end{array}$ & Osteopenia and primary osteoporosis \\
\hline Search strategy until (year) & 2007 & 2012 & 2015 & 2017 \\
\hline Protocol registered & NA & NA & NA & Applied \\
\hline Trial sequential analysis & NA & NA & NA & Applied \\
\hline Outcomes & BMD & BMD & $\begin{array}{l}\text { BMD, bone turnover } \\
\text { markers }\end{array}$ & $\begin{array}{l}\text { BMD, BGP, serum calcium, serum } \\
\text { phosphorus, VAS, CTX, OSC }\end{array}$ \\
\hline
\end{tabular}

Abbreviations: BGP, bone gla protein; BMD, bone mineral density; CTX, C-terminal telo-peptide of type I collagen; NA, not available; OSC, osteocalcin; RCTs, randomized controlled trials; VAS, Visual Analog Scale.

osteoporosis. ${ }^{59}$ Observational studies suggest that lower plasma levels of 25-hydroxy-vitamin D (25[OH]D) are associated with higher risks of osteoporosis ${ }^{60,61}$ and fractures. ${ }^{62}$ Also, calcium and vitamin D supplements have been recommended for older people to prevent fractures by practice guidelines. However, several meta-analyses recently showed that the use of supplements including calcium, vitamin D, or both was not associated with a low risk of fractures among patients with OP ${ }^{63-65}$ Therefore, in light of the need for safe and cost-effective treatments for POP, further research on nonpharmacological therapy treatments is needed.

\section{Conclusion}

In conclusion, there were positive results for TC to benefit for patients with osteopenia and POP. However, due to the low methodological quality and poor reporting quality, current evidence is insufficient. We need more information to firmly establish benefit-harm profile of TC for osteopenia and POP before we accept it as an evidence-based treatment option in our clinical practice. Furthermore, the quality of data must improve greatly if nondrug therapies are to assume a respected place in the contemporary health care.

\section{Acknowledgments}

The authors would like to express their gratitude to Zhi Mao for giving advice about TSA in this manuscript and to Jasper Roebroek for providing language help. This study has received a grant (\#81373885) from the National Natural Science Foundation of China.

\section{Disclosure}

The authors report no conflicts of interest in this work.

\section{References}

1. WHO Scientific Group on the Prevention and Management of Osteoporosis. Prevention and management of osteoporosis: report of a WHO scientific group. Technical Report Series 921. Geneva: World Health Organization; 2003.
2. Sambrook P, CooperC. Osteoporosis. Lancet. 2006;367(9527):2010-2018.

3. Bergland A, Thorsen H, Kåresen R. Effect of exercise on mobility, balance, and health-related quality of life in osteoporotic women with a history of vertebral fracture: a randomized, controlled trial. Osteoporos Int. 2011;22(6):1863-1871.

4. Wilhelm M, Roskovensky G, Emery K. Effect of resistance exercises on function in older adults with osteoporosis or osteopenia: a systematic review. Physiotherapy. 2012;64(4):386-394.

5. National Osteoporosis Foundation. What is osteoporosis and what causes it? Available from: http://nof.org/articles/7. Accessed July 22, 2014.

6. Qaseem A, Forciea MA, McLean RM, Denberg TD; Clinical Guidelines Committee of the American College of Physicians. Treatment of low bone density or osteoporosis to prevent fractures in men and women: a clinical practice guideline update from the American College of Physicians. Ann Intern Med. 2017;166(11):818-839.

7. Cosman F, de Beur SJ, Leboff MS, et al. Clinician's guide to prevention and treatment of osteoporosis. Osteoporos Int. 2014;25(10): 2359-2381.

8. Lems WF, Dreinhöfer KE, Bischoff-Ferrari H. EULAR/EFORT recommendations for management of patients older than 50 years with a fragility fracture and prevention of subsequent fractures. Ann Rheum Dis. 2016;76(5):1-9.

9. Boonen S, Rosenberg E, Claessens F, Vanderschueren D, Papapoulos S. Inhibition of cathepsin $\mathrm{K}$ for treatment of osteoporosis. Curr Osteoporos Rep. 2012;10(1):73-79.

10. Watkins BA. Endocannabinoids, exercise, pain, and a path to health with aging. Mol Aspects Med. 2018;64:68-78.

11. Castrogiovanni P, Trovato FM, Szychlinska MA, Nsir H, Imbesi R, Musumeci G. The importance of physical activity in osteoporosis. From the molecular pathways to the clinical evidence. Histol Histopathol. 2016;31(11):1183-1194.

12. Bergström I, Parini P, Gustafsson SA, Andersson G, Brinck J. Physical training increases osteoprotegerin in postmenopausal women. J Bone Miner Metab. 2012;30(2):202-207.

13. Yuan Y, Chen X, Zhang L, et al. The roles of exercise in bone remodeling and in prevention and treatment of osteoporosis. Prog Biophys Mol Biol. 2016;122(2):122-130.

14. Santos RV, Viana VA, Boscolo RA, et al. Moderate exercise training modulates cytokine profile and sleep in elderly people. Cytokine. 2012; 60(3):731-735.

15. Musumeci G, Loreto C, Leonardi R, et al. The effects of physical activity on apoptosis and lubricin expression in articular cartilage in rats with glucocorticoid-induced osteoporosis. J Bone Miner Metab. 2013;31(3): 274-284.

16. Pichler K, Loreto C, Leonardi R, Reuber T, Weinberg AM, Musumeci G. RANKL is downregulated in bone cells by physical activity (treadmill and vibration stimulation training) in rat with glucocorticoid-induced osteoporosis. Histol Histopathol. 2013;28(9):1185-1196.

17. Musumeci G. The use of vibration as physical exercise and therapy. J Funct Morphol Kinesiol. 2017;2(2):17. 
18. Lauche R, Langhorst J, Dobos G, Cramer H. A systematic review and meta-analysis of Tai chi for osteoarthritis of the knee. Complement Ther Med. 2013;21(4):396-406.

19. Yan JH, Gu WJ, Sun J, Zhang WX, Li BW, Pan L. Efficacy of Tai chi on pain, stiffness and function in patients with osteoarthritis: a metaanalysis. PLoS One. 2013;8(4):e61672.

20. Plummer J. Acupuncture and Tai chi chuan (Chinese shadow boxing): body-mind therapies affecting homeostasis. In: Lau Y, Fowler JP, editors. The Scientific Basis of Traditional Chinese Medicine. Hong Kong: University of Hong Kong Lau; 1982.

21. Frantzis B. The Power of Internal Martial Arts: Combat Secrets of Ba Gua, Tai Chi, and Hsing-I. Berkeley, CA: North Atlantic Books; 1998.

22. Wolf SL, Coogler C, Xu T. Exploring the basis for Tai chi chuan as a therapeutic exercise approach. Arch Phys Med Rehabil. 1997;78(8): 886-892.

23. Chen YW, Hunt MA, Campbell KL, Peill K, Reid WD. The effect of Tai Chi on four chronic conditions-cancer, osteoarthritis, heart failure and chronic obstructive pulmonary disease: a systematic review and meta-analyses. Br J Sports Med. 2016;50(7):397-407.

24. Wayne PM, Berkowitz DL, Litrownik DE, Buring JE, Yeh GY. What do we really know about the safety of tai chi?: A systematic review of adverse event reports in randomized trials. Arch Phys Med Rehabil. 2014;95(12):2470-2483.

25. Wang C, Schmid CH, Fielding RA, et al. Effect of tai chi versus aerobic exercise for fibromyalgia: comparative effectiveness randomized controlled trial. BMJ. 2018;360:k851.

26. Knobloch K, Yoon U, Vogt PM. Preferred reporting items for systematic reviews and meta-analyses (PRISMA) statement and publication bias J Craniomaxillofac Surg. 2011;39(2):91-92.

27. Higgins JPT, Green S, editors. Cochrane Handbook for Systematic Reviews of Interventions version 5.1.0 [updated March 2011]. The Cochrane Collaboration; 2011. Available from: http://handbook cochrane.org/. Accessed July 15, 2016.

28. Kanis JA, Melton LJ, Christiansen C, Johnston CC, Khaltaev N. The diagnosis of osteoporosis. J Bone Miner Res. 1994;9(8):1137-1141.

29. The Osteoporosis Committee of China Gerontological Society. [Chinese Osteoporosis Recommended Diagnostic Criteria (Second Draft)]. Chin J Osteoporos. 2000;1:1-3. Chinese.

30. AACE/ACE Guidelines. American Association of Clinical Endocrinologists and American College of Endocrinology Clinical Practice Guidelines for The Diagnosis and Treatment of Postmenopausal Osteoporosis-2016; 2016. Available from: https://www.aace.com/files/ postmenopausal-guidelines.pdf. Accessed October 6, 2017.

31. Kong J, Wang O, Xing X. Interpretation of the clinician's guide to prevention and treatment of osteoporosis 2014. Drug evaluation. 2015;12(15):8-12.

32. Chinese Society of Bone and Mineral Research. Guideline for diagnosis and treatment of primary osteoporosis. Chin J Osteoporos Bone Miner Res. 2011;1:2-17.

33. Ware JE, Sherbourne CD. The MOS 36-item short-form health survey (SF-36). I. Conceptual framework and item selection. Med Care. 1992; 30(6):473-483.

34. Guyatt GH, Oxman AD, Vist GE, et al. GRADE: an emerging consensus on rating quality of evidence and strength of recommendations. $B M J$. 2008;336(7650):924-926.

35. Furlan AD, Pennick V, Bombardier C, van Tulder M; Editorial Board, Cochrane Back Review Group. 2009 updated method guidelines for systematic reviews in the Cochrane Back Review Group. Spine. 2009; 34(18):1929-1941.

36. Gross A, Miller J, D’Sylva J. Manipulation or mobilisation for neck pain. Cochrane Database Syst Rev. 2010;1:CD004249.

37. Balshem H, Helfand M, Schünemann HJ, et al. GRADE guidelines: 3 . Rating the quality of evidence. J Clin Epidemiol. 2011;64(4):401-406.

38. Holst LB, Petersen MW, Haase N, Perner A, Wetterslev J. Restrictive versus liberal transfusion strategy for red blood cell transfusion: systematic review of randomised trials with meta-analysis and trial sequential analysis. BMJ. 2015;350:h1354.
39. Xia Y, Luo H, Liu JP, Gluud C. Phyllanthus species versus antiviral drugs for chronic hepatitis B virus infection. Cochrane Database Syst Rev. 2013;4:CD009004

40. Zhou Y. Effect of exercise training on prevention and treatment of osteoporosis in lumbar spine of postmenopausal women. Chin $J$ Sports Med. 2003;1:72-75.

41. Chan K, Qin L, Lau M, et al. A randomized, prospective study of the effects of Tai Chi Chun exercise on bone mineral density in postmenopausal women. Arch Phys Med Rehabil. 2004;85(5):717-722.

42. Zhou Y. The effect of traditional sports on bone density of menopause women. J Beijing Sport Univ. 2004;3:354-356.

43. Zhou Y, Li Jing CY. Effects of Taiji pushing hand and calcium supplementation on bone mineral density of menopausal women. Chin J Sports Med. 2005;1:106-108.

44. Ma X, Wang W. Clinical study on prevention of bone loss in elderly male by Taijiquan in 32 cases. Shanxi Tradit Chin Med. 2006;10: 1214-1216.

45. Song H. Effects of Taijiquan exercise on bone density and bone metabolism of primary osteoporosis sufferers. J Phys Educ. 2008;11:106-109.

46. Wang C, Yang Z, Xu L, et al. [Effect of Taichi on bone mineral density of menopause]. Urban Tutor. 2009:140-142. Chinese.

47. Chyu MC, James CR, Sawyer SF, et al. Effects of tai chi exercise on posturography, gait, physical function and quality of life in postmenopausal women with osteopaenia: a randomized clinical study. Clin Rehabil. 2010;24(12):1080-1090.

48. Chen R, Li SC. Effects of Taichi and walking on bone mineral density and bone metabolism in elderly women. J Jilin Inst Phys Educ. 2011;1: $87-89$.

49. Zhang W. Observation on the Therapeutic Effect of Taichi Combined with Calcium D on Primary Osteoporosis. Guangzhou, China: Guangzhou University of Traditional Chinese Medicine; 2011.

50. Wayne PM, Kiel DP, Buring JE, et al. Impact of Tai Chi exercise on multiple fracture-related risk factors in post-menopausal osteopenic women: a pilot pragmatic, randomized trial. BMC Complement Altern Med. 2012;12(1):7-11.

51. Fan L. [Experimental Study of Taijiquan and Brisk Walking Exercise Influence on the Physical Function of the Elderly People]. Shandong Institute of P.E. and Sports, Jinan: 2013. Chinese.

52. Chan X, Kang J. Impacts of Tai Chi as adjuvant therapy on the patients with postmenopausal osteoporosis. Chin J Mod Nurs. 2015;31: 3729-3732.

53. Zhao M, Li N, Chao F, et al. Effect of Taiji pushing hand and nutrition intervention on rehabilitation of patients with primary osteoporosis. Liaoning Sport Sci Technol. 2015;3:44-46.

54. Yao C, Wang C, Wang G. The influence of Taichi on the health status of middle-aged and aged women. Chin J Health Care Med. 2016;6:494-496.

55. Bertozzi L, Gardenghi I, Turoni F, et al. Effect of therapeutic exercise on pain and disability in the management of chronic nonspecific neck pain: systematic review and meta-analysis of randomized trials. Phys Ther. 2013;93(8):1026-1036.

56. Lee MS, Pittler MH, Shin BC, Ernst E. Tai chi for osteoporosis: a systematic review. Osteoporos Int. 2008;19(2):139-146.

57. Xu S, Liu P. A meta-analysis of the effect of Taichi on bone mineral density in postmenopausal women. Chin J Osteoporos. 2012;10:932-936.

58. Sun Z, Chen H, Berger MR, Zhang L, Guo H, Huang Y. Effects of tai chi exercise on bone health in perimenopausal and postmenopausal women: a systematic review and meta-analysis. Osteoporos Int. 2016;27(10):2901-2911.

59. Aggarwal S, Nityanand. Calcium and vitamin D in post menopausal women. Indian J Endocrinol Metab. 2013;17(9):618.

60. Bischoff-Ferrari HA, Kiel DP, Dawson-Hughes B, et al. Dietary calcium and serum 25-hydroxyvitamin D status in relation to BMD among U.S. adults. J Bone Miner Res. 2009;24(5):935-942.

61. Kuchuk NO, van Schoor NM, Pluijm SM, Chines A, Lips P, Status VD. Vitamin D status, parathyroid function, bone turnover, and BMD in postmenopausal women with osteoporosis: global perspective. $J$ Bone Miner Res. 2009;24(4):693-701. 
62. Khaw KT, Luben R, Wareham N. Serum 25-hydroxyvitamin D, mortality, and incident cardiovascular disease, respiratory disease, cancers, and fractures: a 13-y prospective population study. Am J Clin Nutr. 2014; 100(5):1361-1370.

63. Zhao JG, Zeng XT, Wang J, Liu L. Association between calcium or vitamin D supplementation and fracture incidence in communitydwelling older adults: a systematic review and meta-analysis. JAMA. 2017;318(24):2466-2482.
64. Kahwati LC, Weber RP, Pan H, et al. Vitamin D, calcium, or combined supplementation for the primary prevention of fractures in communitydwelling adults: evidence report and systematic review for the US preventive services task force. JAMA. 2018;319(15):1600-1612.

65. Bolland MJ, Grey A, Avenell A. Effects of vitamin D supplementation on musculoskeletal health: a systematic review, meta-analysis, and trial sequential analysis. Lancet Diabetes Endocrinol. 2018;6(11):847-858.

\section{Publish your work in this journal}

Clinical Interventions in Aging is an international, peer-reviewed journal focusing on evidence-based reports on the value or lack thereof of treatments intended to prevent or delay the onset of maladaptive correlates of aging in human beings. This journal is indexed on PubMed Central, MedLine,

\section{Dovepress}

CAS, Scopus and the Elsevier Bibliographic databases. The manuscript management system is completely online and includes a very quick and fair peer-review system, which is all easy to use. Visit http://www.dovepress. com/testimonials.php to read real quotes from published authors. 